Capuani Silvia (Orcid ID: 0000-0002-7863-1801)

\title{
High-resolution multiparametric MRI of contemporary and waterlogged archaeological wood
}

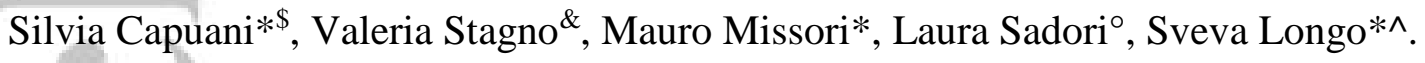

*National Research Council - Institute for Complex Systems (CNR-ISC) c/o Physics

Department Sapienza University of Rome, Rome, Italy.

${ }^{\$}$ Centro Fermi - Museo Storico della Fisica e Centro Studi e Ricerche Enrico Fermi

Piazza del Viminale 1, 00184 Rome, Italy

${ }^{\&}$ Department of Earth Sciences, Sapienza University of Rome, Piazzale Aldo Moro 5, 00185 Rome, Italy.

' Department of Environmental Biology, Sapienza University of Rome, Piazzale Aldo Moro 5, 00185 Rome, Italy.

${ }^{\wedge}$ Department of Mathematical and Computational Sciences, Physics Science and Earth Sciences (MIFT), University of Messina, Messina 98166, Italy.

\section{Abstract}

High-resolution NMR images on three different present-day wood samples and an archaeological wood specimen were presented and discussed. Although the spatial resolution is still low to perform dendrology for the exact identification of the wooden species, the $T_{2}$ spin-density weighted images exhibit contrasts that are in excellent agreement with optical histological images. On the other hand, $\mathrm{T}_{2} *$ and $\mathrm{T}_{1}$-weighted images provide physiological information that is not obtainable by the usual light microscopic investigations. Moreover, the diffusion-weighted images show the anisotropic behavior of the water diffusion coefficient quantified along and perpendicularly to vascular bundles (vessels and tracheids), which can be related to the morphology and size of wooden microstructure. This work suggests that high-resolution multiparametric MRI may be a useful tool to increase the information obtainable from the waterlogged archaeological wood remains in a completely non-invasive and non-destructive approach. Therefore, it would be desirable to further develop the hardware and functional characteristics of MRI scanners to improve their potential application in the field of wooden cultural heritage.

This article has been accepted for publication and undergone full peer review but has not been through the copyediting, typesetting, pagination and proofreading process which may lead to differences between this version and the Version of Record. Please cite this article as doi: $10.1002 / \mathrm{mrc} .5034$ 
Keywords:

MRI, ${ }^{1} \mathrm{H}$, diffusion, microimaging, microstructure, archaeological wood, waterlogged wood, poplar, stone pine, spruce.

Abbreviations

MRI Magnetic Resonance Imaging

Introduction

Magnetic Resonance Imaging (MRI), i.e. the spatially resolved NMR, is known to be a powerful clinical investigation method that allows the observation of the internal anatomy of human tissues through images representative of the spatial distribution of mobile protons (mainly from water and fat). Thanks to the exquisite sensitivity of the observed proton spins to the surrounding environments, the quantification of protons relaxation times $\mathrm{T}_{1}, \mathrm{~T}_{2}$ and $\mathrm{T}_{2}$ * and molecular diffusion D make possible to probe the physico-chemical and microstructural properties of tissues ${ }^{[1,2]}$. Importantly, MRI is a multiparametric investigation: in the NMR images, in addition to the anatomical information, the information deriving from one of the aforementioned parameters selected using a specific acquisition sequence is superimposed $^{[1,2]}$. This incredibly increases the effectiveness of clinical diagnostics ${ }^{[1,2]}$. Despite MRI is a very promising and attractive method for providing detailed, non-invasive, and quantitative information in living materials containing water, MRI in wood research is still far from being a routine tool ${ }^{[3,4]}$. However, wood is an ideal material for MRI studies, due to its high moisture content when green and its hygroscopic character when in use. Wood is a natural heterogeneous and anisotropic porous material constituted by a solid matrix with pores characterized by diameter between 0-400 microns. In particular, each wooden species has structures such as: vessels, tracheids, fibers, pits, perforations (see Fig. 1) with peculiar dimensions, morphological features, and spatial organizations. Wood is classified as softwood and hardwood (Fig. 1). In softwood species the wood cells are mostly of one kind, named tracheids, and as a result the material is much more uniform in structure than that most hardwoods. In hardwood the water conducting capability is mostly taken care of by vessels that are in some cases quite large and distinct, in others too small to be seen without a hand lens. The expected quality of MRI improves as the moisture content of the wood increases. In fact, as the quantity of mobile protons increases, the signal to noise ratio (SNR) of the images increases, a necessary condition for enhancing the spatial resolution, which depends on the magnetic field gradients strength used to obtain spatially resolved NMR information $^{[5,6]}$. 
In this regard, the archaeologically excavated wood generally has a very high moisture content (400\%-800\%) thus proving to be an ideal investigative subject for high-resolution NMR imaging.

In particular, waterlogged archaeological wood is very well maintained if left immersed, while it undergoes rapid deterioration once extracted from the water. Currently, the recognition of the wooden species takes place through the destructive histological examination which involves the cutting of thin slices of wood to be observed under an optical microscope. Non-invasive methods based on x-ray images have recently been developed allowing non-invasive recognition by microscopic examination of at least three rings ${ }^{[7]}$. However, the latter method seems to fail in the case of waterlogged wood, due to the excessive water content which attenuates the contrast obtained with x-ray imaging of the different wooden structures soaked in water ${ }^{[7]}$.

Another useful property of NMR imaging is of being able to monitor chemical-physical processes in a non-invasive way. MRI may monitor both consolidation and extraction procedures (with consequent identification of extracted elements) of waterlogged archaeological wood. Indeed, relaxation times, especially $\mathrm{T}_{2}{ }^{[5,6]}$, are iron-sensitive. Therefore $\mathrm{T}_{2}{ }^{*}$-weighted MRI measurements could be used to identify iron-contaminated wood samples ${ }^{[8,9]}$. Regarding the consolidation procedures, the only way to save waterlogged wood is its proper conservation immediately after excavation from the wet environment. This involves the exchange of the water that fills the wood tissue for an appropriate chemical that will strengthen the wood structure and prevent its distortion, but unlike water, it will not evaporate from the wood when exposed to air ${ }^{[10]}$. Therefore, studies on conservation agents that meet such requirements are desirable in order to better preserve the wooden remains. For this purpose, it is possible to monitor by MRI the flow of chemical agents that replace the water in the wood and investigate their interaction with the wood structures. ${ }^{[10,11]}$ In this paper, we show that high-resolution MRI can be a useful tool for the identification of waterlogged wood species and to assess their condition and conservation.

Towards this goal, high-resolution NMR images performed at 9.4T on three different presentday wood samples (a hardwood and two softwood species) imbibed with water and a waterlogged archeological wood specimen were presented and discussed. In particular multiparametric MRI was performed to achieve images along the three different anatomical wood planes (see Fig. 1) for obtaining a 3D virtual histology of the aforementioned samples. The in-plane resolution was around $9 \times 9 \mu \mathrm{m}^{2}$ and $20 \times 20 \mu \mathrm{m}^{2}$ depending on the different NMR parameters selected in the weighted image. 


\section{Materials and methods}

\section{Wood samples}

Four different wood samples in total were investigated: a) white poplar (Populus alba $\left.)^{[12,13,14]}, \mathrm{b}\right)$ stone pine (Pinus pinea $\left.)^{[13-15]}, \mathrm{c}\right)$ spruce $\left(\right.$ Picea abies) ${ }^{[13-15]}, \mathrm{d}$ ) archeological wood extracted from a pole belonging to the old Roman port of Naples ( $5^{\text {th }}$ century AD) that was found during the underground excavations at Piazza Municipio (Naples, Italy). White poplar was chosen because it was widespread since ancient times in Central and Southern Europe and Northern Africa but above all because of its diffuse-porous hardwood ${ }^{[13-15]}$, consisting of vessels and different water compartments and microstructures, besides fibers. The softwood ${ }^{[13-15]}$ of pine and spruce, present in Italy and widely used already 2000 years ago, are selected as candidates for the recognition of the archaeological wood sample classified as softwoods.

Wood samples were shaped obtaining five cylinders-like blocks of about $1.2 \mathrm{~cm}$ in height and $0.7 \mathrm{~cm}$ in diameter. The cut was made so that the height of the cylinder was parallel to the longitudinal directions, i.e. to the main direction of the vessels/tracheids (see Fig. 1). To perform NMR experiments, each sample (with the exception of the archaeological wood sample) was immersed in distilled water until the saturation was reached. Each sample was inserted in NMR tubes and water to perform MRI measurements. Once the wood samples were put in the magnetic field, their longitudinal direction, i.e. the direction parallel to the longest axis of the vessels/samples, was parallel to the direction of the static magnetic field $\mathrm{B}_{0}$, conventionally along $\mathrm{z}$ axis (Fig. 1). Fig.1 summarizes the main observation directions of wood anatomy together with the direction of the static magnetic field, the $\mathrm{x}$ and $\mathrm{z}$ axes of the laboratory reference frame and the axial-view plane that allows observing the cross-sections of the vessels/tracheids.

\section{MRI}

Micro-magnetic resonance imaging investigations were performed using a Bruker Avance400 spectrometer operating at $9.4 \mathrm{~T}$ with a $10 \mathrm{~mm}$ micro-imaging probe equipped with high performance and high strength magnetic field gradient unit characterized by a maximum gradient strength of $1200 \mathrm{mT} / \mathrm{m}$ and a rise time of $100 \mu \mathrm{s}$. ParaVision ${ }^{\circledR} 3.2$ software was employed for data acquisition and image processing.

A multi-slice multi-echo (MSME) sequence with minimum echo time (TE) of $2.1 \mathrm{~ms}$, repetition times (TR) equal to $350 \mathrm{~ms}$ and 64 signal averages (NSA) was used to obtain $\mathrm{T}_{1-}$ 
weighted images. Six slices of $400 \mu \mathrm{m}$ in thickness (STK) were selected, field of view (FOV) was $0.70 \mathrm{~cm}$, imaging matrix (MTX) equal to $256 \times 256$, or $512 \times 512$ voxels.

MSME with $T R=1 \mathrm{~s}$ and different $\mathrm{TE}$ was used to obtain $\mathrm{T}_{2}$-weighted images at about $20 \times 20$ $\mu \mathrm{m}^{2}$ in plane resolution were acquired with acquisition parameters optimized for each wood species.

A gradient echo fast imaging (GEFI) sequence with $\mathrm{TR}=1 \mathrm{~s}, \mathrm{TE}=4 \mathrm{~ms}, \mathrm{STK}=300 \mu \mathrm{m}$, $\mathrm{FOV}=0.9 \mathrm{~cm}$, matrix $=512 \times 512$ voxels, $\mathrm{NS}=128$ was adopted to achieve axial $\mathrm{T}_{2}{ }^{*}$-weighted images and with the same parameters but with $\mathrm{TR}=1.5 \mathrm{~s}$ and $\mathrm{TE}=5 \mathrm{~ms}$ to obtain radial and tangential view $\mathrm{T}_{2} *$-weighted images. Moreover, to improve image resolution, acquisition matrices of $1024 \times 1024$ voxels were also used by selecting TE=6 ms.

An imaging Pulse Gradient Stimulated Echo (PGSTE) sequence was used to investigate water diffusion in wood samples. The sequence parameters were: TR=1.5 s, TE $=14.8 \mathrm{ms,}$ matrix 256x256 voxels, $\mathrm{FOV}=0.8 \mathrm{~cm}$, diffusion gradient pulse duration $\delta=3 \mathrm{~ms}$, diffusion time $\Delta=80 \mathrm{~ms}$. The diffusion gradient was used both in $\mathrm{z}$ and $\mathrm{x}$-direction, i.e. along and perpendicular to the long axis of wooden vessels/tracheids, respectively. The effective bvalues were approximately equal to: $260,960,1260,2060 \mathrm{~s} / \mathrm{mm}^{2}$.

\section{Optical investigation}

Size calibrated pictures of thin sections of wood samples were obtained in transmission mode by using a stereo microscope STMPRO-T BEL Engineering (Italy) equipped with a EUREKAM 3.0 3MPixel camera. BEL View 7 software was used for image acquisition.

\section{Results and discussion}

Since MRI is a fundamental technique for medical diagnostics, the selection of observation plans of any object to perform NMR imaging follows the three primary imaging planes that are utilized in neuroimaging ${ }^{[16]}$. However, to perform the virtual histology of wood by MRI it is important to obtain images by selecting slices along well-defined wood anatomical sections (planes) ${ }^{[13-15]}$. As a consequence, care should be taken in referring to the orientation of the NMR image plans when a wood sample is investigated. Therefore, as underlined in Fig. 1, the transversal section (view plane) used in the description of the wood anatomy coincides with the axial-view plane of the medical imaging. Specifically, the transversal plane of view is the plane perpendicular to vessels/tracheids main axis, by which crosssection of vessels/tracheids can be observed and measured. 
An axial view $\mathrm{T}_{2} *$-weighted image of water imbibed white poplar, characterized by an inplane resolution of $18 \times 18 \mu \mathrm{m}^{2}$ and $\mathrm{STK}=300 \mu \mathrm{m}$, is displayed in Fig. 2a. In Fig. 2b a zoomed portion of the image is reported with some measurement of the wood structure. Hyperintense white pores are the cross-section of vessels. Their size runs from 20 to 80 microns in diameter. Image contrast is due to lower $\mathrm{T}_{2} *$ values associated with parenchyma structures (intracellular water) and higher $\mathrm{T}_{2} *$ values associated with larger pores. In Fig. $2 \mathrm{c}$ and Fig. 2d, MRI and conventional optical image are shown, respectively. By comparing the images shown at the same resolution it is possible to ascertain the agreement in wood structure details between the conventional optical histology and the MRI virtual histology. As much as conventional optical histology, the $\mathrm{T}_{2}{ }^{*}$-weighted image shows the presence of measurable microstructures such as rings and rays highlighted by arrows in Fig. 2. On the other hand, unlike optical investigations, MRI allows a non-destructive volumetric evaluation of the whole sample microstructures along different planes of observation (transversal, tangential and radial) as shown in Fig. 3 and therefore it provides a better overview of the topology and complex rearrangement of the wooden structures. Moreover, multiparametric MRI provides multiple structural and functional information that allows obtaining more information than just the structural information provided by optical imaging. In this regard, $\mathrm{T}_{2}$-weighted and $\mathrm{T}_{1}$-weighted images of a waterlogged archaeological wood specimen are displayed in Fig. 4. By observing and comparing the two images in Fig. 4, it is possible to discriminate microstructures characterized by different $T_{1}$ and $T_{2}$ contrasts that provide valuable information about the dynamics and surroundings of the microstructures. Indeed, the different single parameter image gives a better insight into the morphology of wood anatomy. In particular, it is immediately possible to conclude that the unknown archaeological wood specimen is a softwood species. Indeed, according to Kekkonen et al. results ${ }^{[17,18]}$ the softwood is characterized by higher and lower signal intensity zones in $\mathrm{T}_{1}$ and $\mathrm{T}_{2}$-weighted images, representing earlywood and latewood areas, respectively. Image contrast is due to lower $\mathrm{T}_{2}$ values associated with smaller pores (cross-sectional areas of tracheids) in latewood and higher $\mathrm{T}_{2}$ values associated with larger pores in earlywood.

The images also show the presence of artifacts due to air bubbles and/or the presence of paramagnetic agents. As expected, such artifacts are amplified in the $\mathrm{T}_{2}{ }^{*}$-weighted images of the same archaeological wood specimen shown in Fig. 5. Here, images with an in-plane resolution of $18 \times 18 \mu \mathrm{m}^{2}$ and $300 \mu \mathrm{m}$ of slice thickness and obtained along the three principal anatomical planes of wood are displayed. Different kinds of microstructure are recognizable 
and highlighted with arrows of different colors in the images (see Fig. 5). The unknown archaeological wood has also resin channels (see the zoomed image in Fig. 5b) that appear as large (larger than the cross-section of tracheids) white circles surrounded by a thin darker ring due to parenchyma cells that delimit the resin canal (red arrows in Fig. 5a). In Fig. 5c) unicellular rays comprised of a few cells and with resin canal (red arrow) are also observable. Not all the softwoods have large resin canals in the tangential section, therefore this observable microstructure is a potential identification key to identify the wood species of the archaeological wood sample. Even the identification of the number of cells in rays is a potential identification key to recognize a wood species. However, the impossibility to distinguish, by current MRI, the type of cells (another key anatomical feature) composing rays has to be pointed out. Parallel to the great potential of MRI for the non-invasive investigation of waterlogged wood, the limits of the technique are principally related to the insufficient MRI resolution compared to the optical one when the wood radial section is examined. In this work (Fig. 5c) the diagnostic features of wood, such as the walls and pits of cross-fields and the presence of radial tracheids, are not visible due to their small size (lower than $10 \mu \mathrm{m})$ and the specific image-contrast selected. However, the linear NMR image resolution below $10 \mu \mathrm{m}$ is not a physics limit, but it is due to the specific hardware and software of the spectrometer. Since the resolution of an NMR image depends on the magnetic field gradients strength, in principle it is possible, using higher gradients strength than those used in this work, to obtain images with a resolution around the micrometer. However, a good signal to noise ratio (SNR) of the image must be ensured, which decreases with increasing resolution. To increase the SNR it is possible to increase the number of signal averages, or to increase the static magnetic field strength. ${ }^{[5,6,19]}$

In Fig.6 and Fig.7, high-resolution $\mathrm{T}_{2}{ }^{*}$-weighted MRI on Pine and Spruce are displayed, respectively. From the first examination of Fig. 6, the wooden structure appears very similar to that of the archaeological wood sample (Figs.4 and Fig.5). However, for precise recognition of the wood species, it is necessary to observe the microstructures present in the tangential and radial anatomical planes. Moreover, as previously pointed out, the slice thickness of 300 or $200 \mu \mathrm{m}$ of tangential and radial images does not allow a good spatial resolution. Furthermore, the in-plane resolution is still insufficient to observe microstructures smaller than $10 \mu \mathrm{m}$ such as the pits, and their type, distribution and density along vessels and in the cross-field. Moreover, scalariform or simple perforations, vessels with and without thickening, homogeneous or inhomogeneous rays, the punctuation of radius and vessels and 
the cross-fields in the radial section ${ }^{[13-15]}$ are not still visible. In this regard, diffusionweighted MRI methods can indirectly determine the size and density of microstructures, regardless of the image resolution, or, in other words, independently from the size of the image voxel. In fact, NMR images weighed in the molecular diffusion of water are characterized by an intrinsic resolution related to the diffusion length $l_{D}$ through which the system is probed ${ }^{[5,20-22]}$. The possibility of assessing the size of cells in wood through molecular diffusion measurement of water in the wooden tissue was highlighted some years ago, considering cells of only one size ${ }^{[23]}$. Currently, with the development of new methods and models of molecular diffusion quantified with NMR, it is possible to obtain good results also for determining the size of different types of cells and microstructures, their orientation and their shape ${ }^{[5,20,21]}$.

In Fig. 8 axial-view diffusion-weighted images of Spruce are displayed. The upper row shows the diffusion-weighted images obtained with the magnetic field gradient $\mathrm{g}$ along the $\mathrm{x}$ direction, while the second row shows the images obtained with g along the z-direction. Since in the tracheids water diffuses more easily along the z-direction, i.e. along the main axes of the tracheids (see Fig. 1) the diffusion coefficient D of water inside the tracheids assumes higher values along the $\mathrm{z}$ direction $\left(\mathrm{D}=(1.7 \pm 0.3) * 10^{-3} \mathrm{~mm}^{2} / \mathrm{s}\right)$ rather than along the $\mathrm{x}$ direction $\left(\mathrm{D}=(0.83 \pm 0.01) * 10^{-3} \mathrm{~mm}^{2} / \mathrm{s}\right)$ showing anisotropic diffusion. Moreover, mean value of the diffusion coefficient are different in earlywood $\left(\mathrm{D}=(0.83 \pm 0.01) * 10^{-3} \mathrm{~mm}^{2} / \mathrm{s}\right.$ along $\mathrm{X}-$ direction) and latewood $\left(\mathrm{D}=(0.43 \pm 0.02)^{*} 10^{-3} \mathrm{~mm}^{2} / \mathrm{s}\right)$. Earlywood and latewood are highlighted in Fig. 8 with red and yellow circles, respectively. These results are in agreement with the lower size of tracheids cross-sections in latewood compared to earlywood tracheids size.

In conclusion, this work suggests that high-resolution multiparametric MRI may be a useful tool to obtain physiological and microstructural information from both full water imbibed wood samples and waterlogged archaeological wood remains in a completely non-invasive and non-destructive approach. This is particularly important especially when the mechanical sectioning necessary for the optical investigation is not easy to perform due to the fragility and decay of the wood remains. In this case, virtual histology can be a valid alternative to optical microscopy. The major limit to the development of dendrology performed using 3D virtual histology based on MRI concerns the size of the image voxel, still too large compared to fundamental microstructures for the discrimination of similar types of wood. However, this is a limit related to the instrument, it is not a physical limit. Therefore, it would be desirable 
to further develop the hardware and functional characteristics of MRI scanners to improve their potential application in the field of wooden cultural heritage.

\section{References}

[1] P. Tofts, Quantitative MRI of the brain, Wiley, West Sussex, UK, 2003.

[2] T.H. Berquist, MRI of musculoskeletal System, Lippincott Williams \& Wilkins, Philadelphia, 2006.

[3] L.D. Hall, V. Rajanayagam, Wood Sci. Technol. 1986, 20, 329.

[4] S.V. Dvinskikh, M. Henriksson, L.A. Berglund, I. Furó, Holzforschung, 2011, 65, 103.

[5] P.T. Callaghan, Principles of Nuclear Magnetic Resonance Microscopy, Oxford University Press Inc, New York, 1991.

[6] P. Mansfield, NMR Imaging in Biomedicine: Supplement 2 Advances in Magnetic Resonance, Elsevier, 1982

[7] M. Mori, S. Kuhara, K. Kobayashi, S. Suzuki, M. Yamada, A. Senoo, Dendrochronologia 2019, 57, 125630.

[8] D.I. Cole-Hamilton, B. Kaye, I.A. Chudek, G. Hunter, Studies in Conservation 1995, 40, 41.

[9] Y. Kanazawa, T. Yamada, A. Kido, K. Fujimoto, K. Takakura, H. Hayashi, Y. Fushimi, S. Kozawa, K. Koizumi, M. Okuni, N. Ueda, K. Togashi 2017 Magn Reson Imaging 38, 196.

[10] J. Kowalczuk, A. Rachocki, M. Broda, B. Mazela, G.A. Ormondroyd, J. Tritt-Goc, Wood Sci Technol 2019, 53, 1207.

[11] DW Grattan 1982 Stud Conserv 27:24-136.

[12] A. Alesiani, F. Proietti, S. Capuani, M. Paci, M. Fioravanti, B. Maraviglia, Appl Magn Reson 200529 (2), 177.

[13] M. Plötze, P. Niemz, Eur. J. Wood Prod., 2011 69, 649.

[14] H.G. Richter, D. Grosser, I. Heinz, P.E. Gasson, IAWA J., 2004, 25, 1.

[15] P.O. Kettunen, Wood Structure and Properties, Trans Tech Publications, Ltd., 2006.

[16] P.C. Lebby, Brain Imaging: a guide for clinicians, Oxford University Press, 2013

[17] P.M. Kekkonen, V.-V. Telkki, J. Jokisaari, J. Phys. Chem. B, 2009, 1131080.

[18] P.M. Kekkonen, V.-V. Telkki, J. Jokisaari, J. Phys. Chem. C, 2010, 114, 18693.

[19] D. Di Nardo, G. Gambarini, S. Capuani, L. Testarelli J. Endod. 2018, 44, 536.

[20] S. Capuani, G. Manenti, R. Iundusi, U. Tarantino, BioMed Research International 2015 Article ID 948610. 
[21] S. Capuani, M. Palombo, Frontiers in Physics, 2019, 7, 248.

[22] R. Valiullin, V. Skirda, Journal of Chemical Physics 2001,114, 452.

[23] W. Wycoff, S. Pickup, B. Cutter, W. Miller, T.C. Wong, Wood and Fiber Science 2000, 32,72 .

\section{ACKNOWLEDGEMENTS}

We acknowledge funding of Regione Lazio under the ADAMO project $\mathrm{n}$. B86C18001220002 of the Centre of Excellence at the Technological District for Cultural Heritage of Lazio (DTC).

The authors would like to thank the Istituto Centrale del Restauro (ICR) of Rome (Italy) for providing the archaeological wood sample.

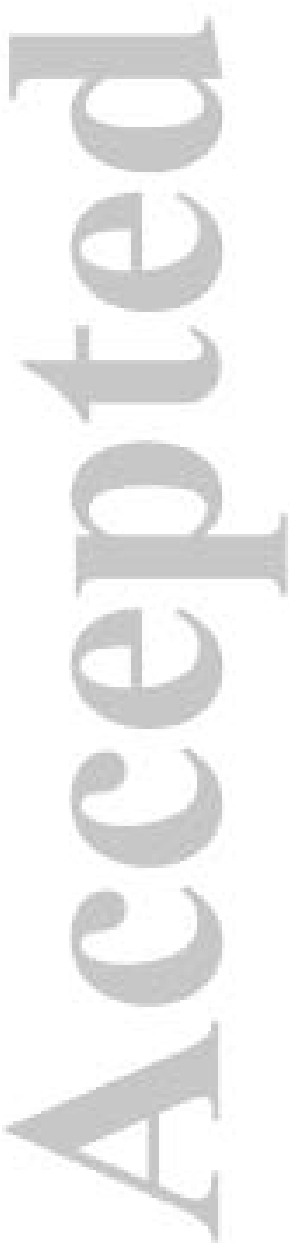




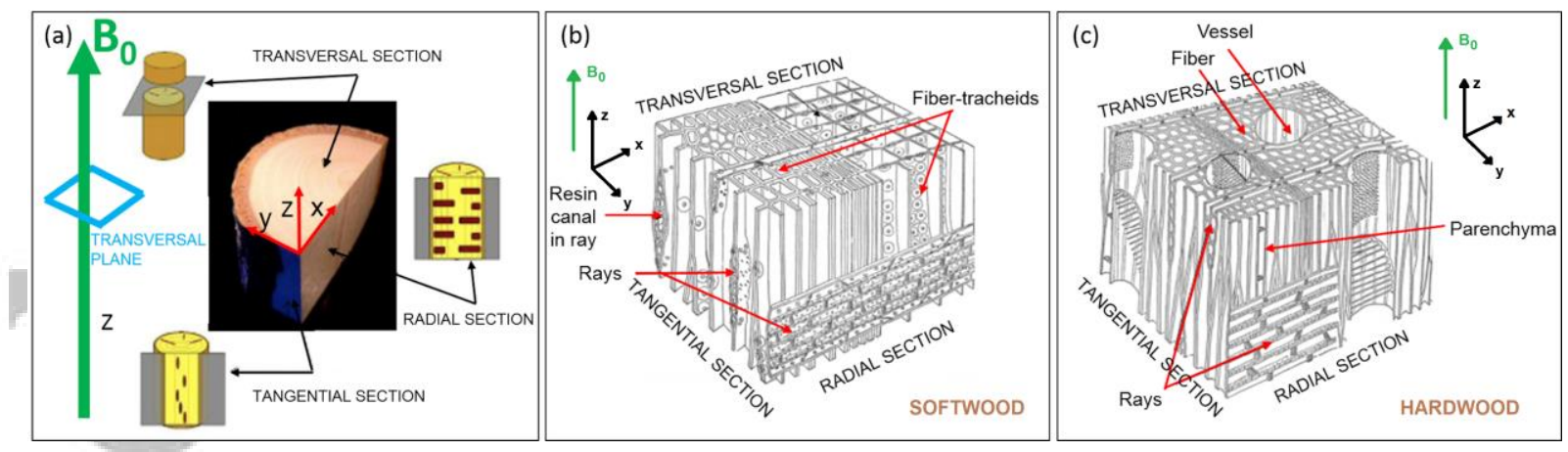

Fig.1 (a) Schematic representation of the main directions for observing wood anatomy (transverse and key longitudinal sections, i.e. tangential, radial sections) compared with the direction of the static magnetic field $\mathrm{B}_{0}$ along the $\mathrm{z}$-axis; the $\mathrm{x}$ and $\mathrm{z}$-axis of the laboratory reference frame and the axial (transversal) view plane that allows to observe and measure the cross-sections of the vessels/tracheids. Sketches of (b) softwood and (c) hardwood with key anatomical features. 

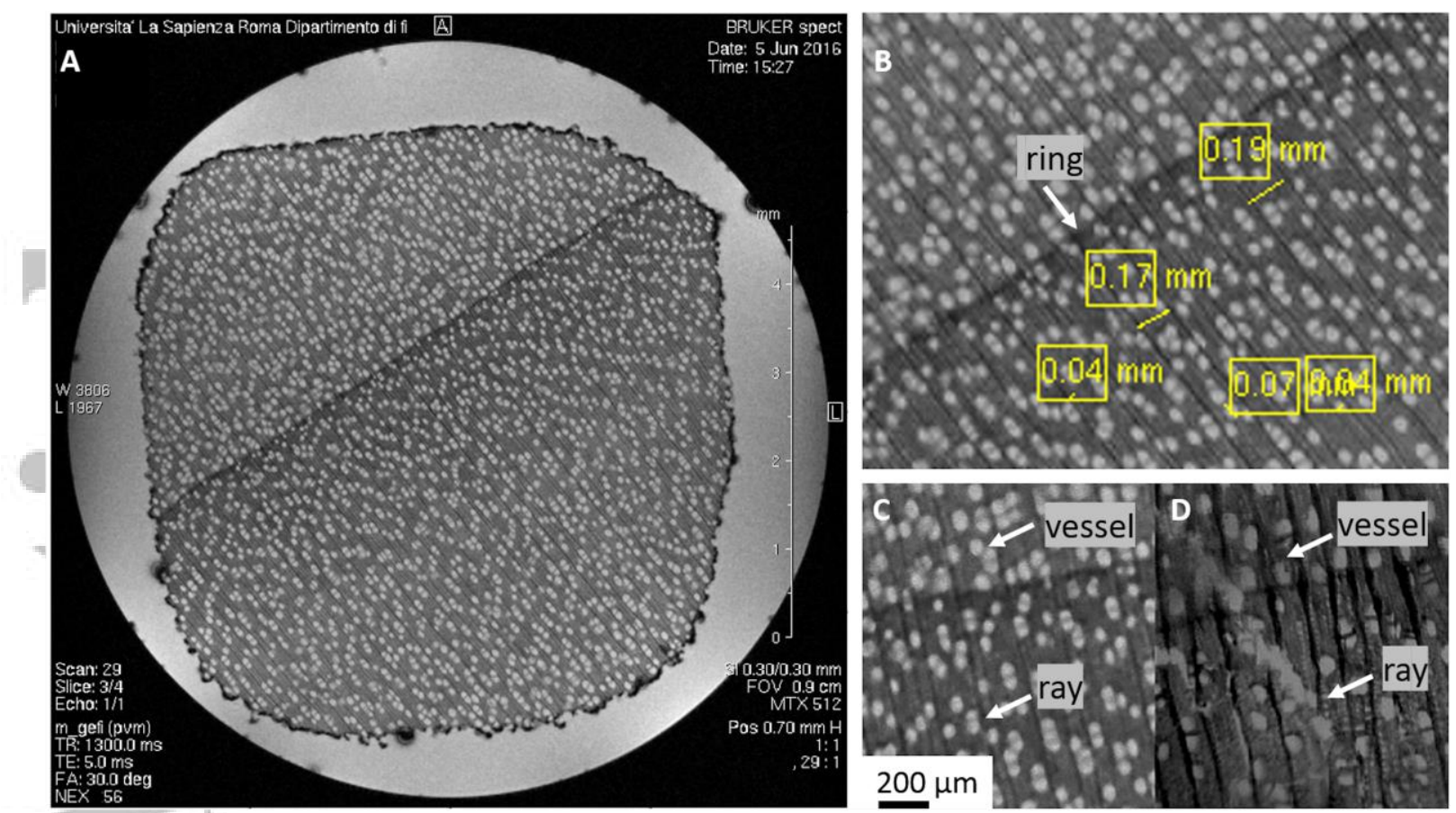

Fig. 2 Axial-view $\mathrm{T}_{2}{ }^{*}$-weighted image of water imbibed White Poplar, characterized by an in-plane resolution of $18 \times 18 \mu \mathrm{m}^{2}$ and STK=300 $\mu \mathrm{m}$ (2a). Zoomed portion of the image in a) with some measurement of wood structure: rays mean distance is about $170-180 \mu \mathrm{m}$, mean cross-section vessel size is in the range 20-80 $\mu \mathrm{m}$. The vessels are distributed singly or in pairs, triples and quadruple (2b). MRI (2c) and Optical image (2d) of the same White Poplar specimen. As much as conventional optical histology, the $\mathrm{T}_{2}{ }^{*}$-weighted image shows the presence of measurable microstructures such as rings, rays and libriform fibers (highlighted

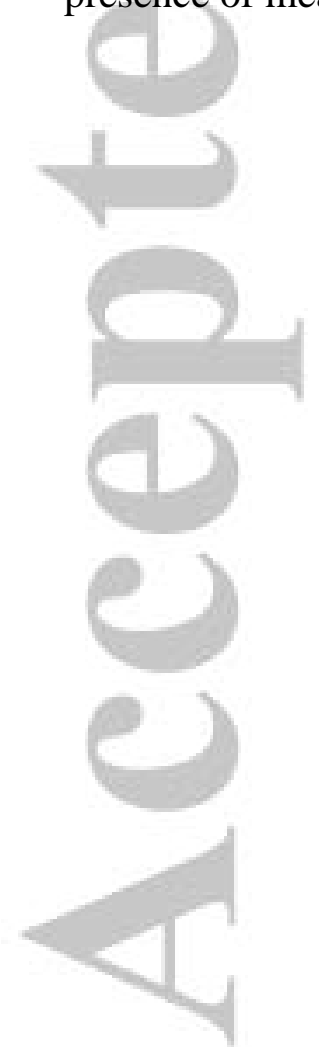
by arrows) 

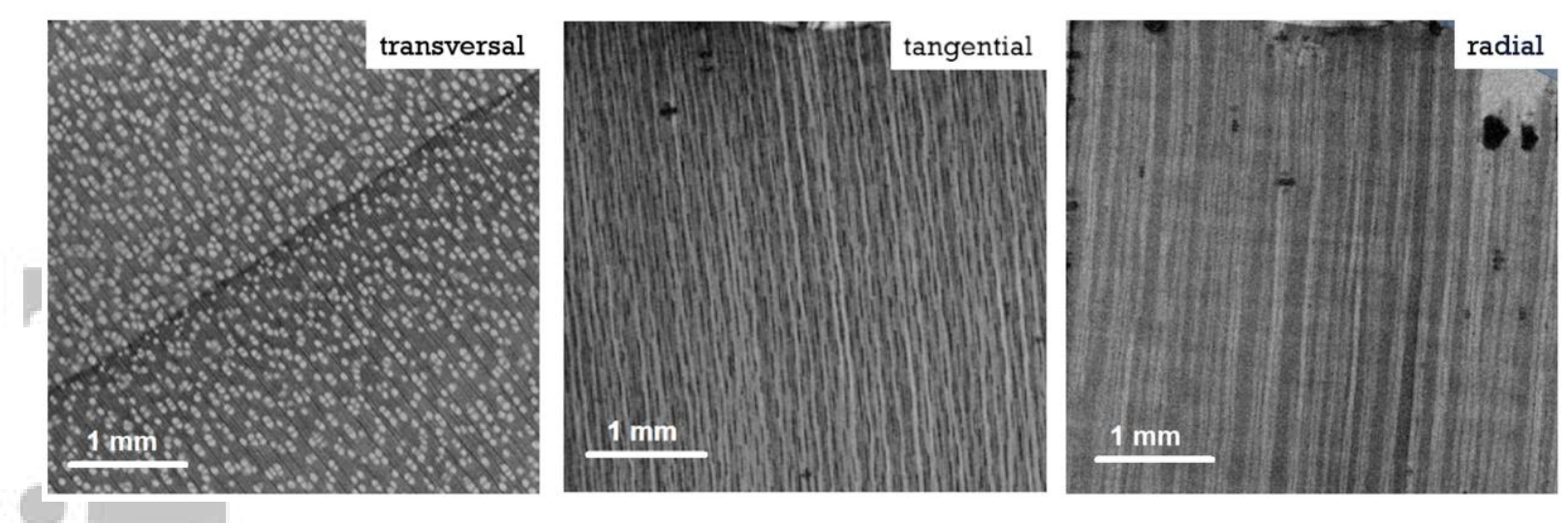

Fig. 3 Transversal a), tangential b) and radial c) -view MRI of water imbibed White Poplar sample which provides a complete virtual 3D histology of Poplar. Using an in-plane resolution of $18 \times 18 \mu \mathrm{m}^{2}$ and $300 \mu \mathrm{m}$ of slice thickness, vessels and parenchyma are well visible in the transversal-view image. Vessels (light grey voxels) and fibers (darker voxels) in tangential and radial view are visible but it would be necessary to select a smaller slice

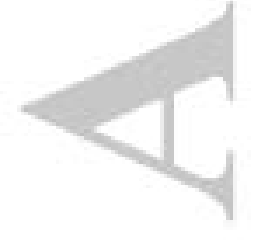
thickness to better resolve them spatially.

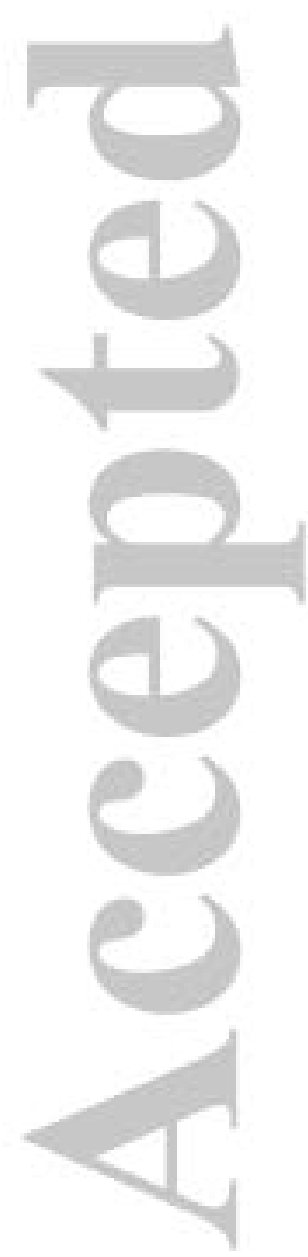



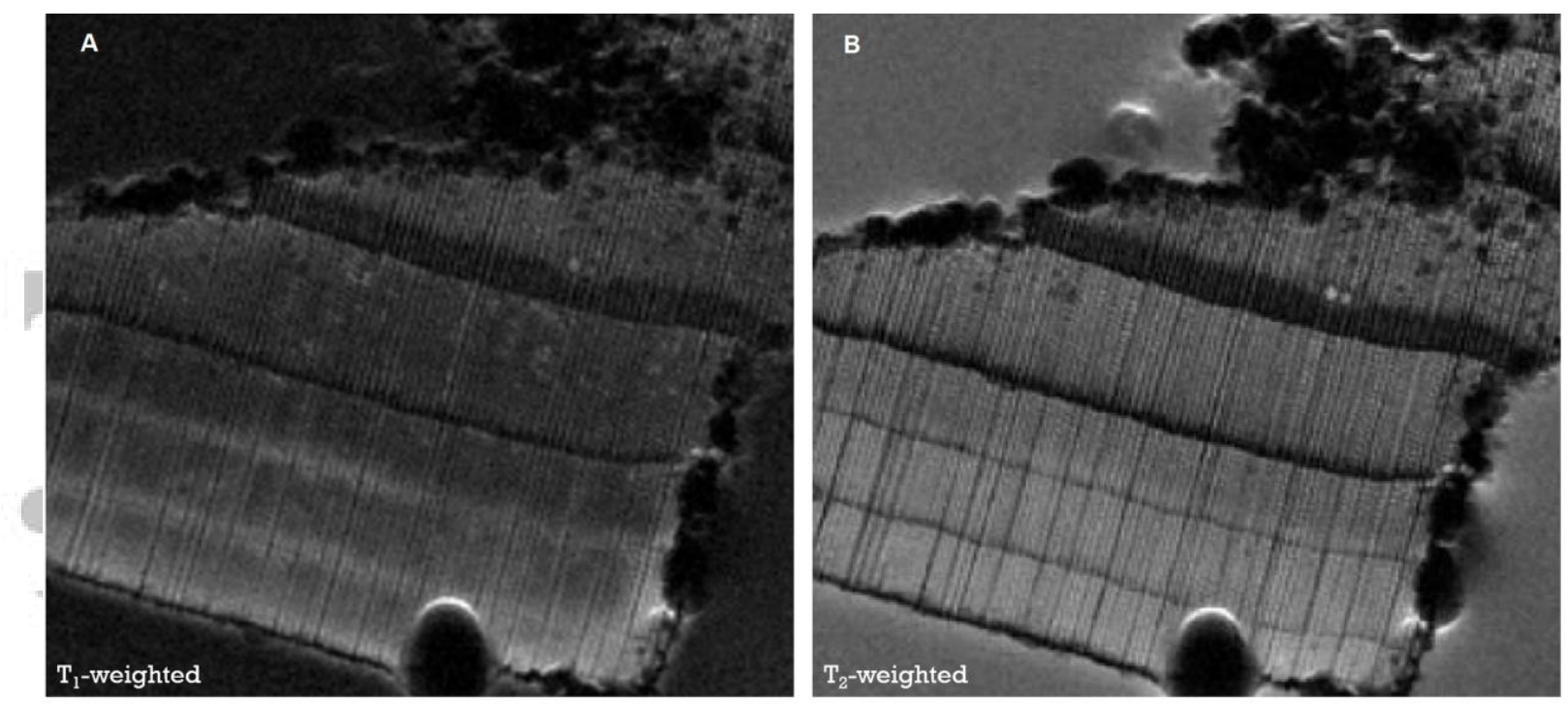

Fig. 4 Transversal-view MRI of an archaeological waterlogged wood specimen (from an ancient port in Naples of the 5th century AD): $\mathrm{T}_{1}$-weighted image with $\mathrm{TE}=2 \mathrm{~ms}$ and $\mathrm{TR}=350$ $\mathrm{ms}$ (left) and $\mathrm{T}_{2}$-weighted image with $\mathrm{TR}=1000 \mathrm{~ms}$ and $\mathrm{TE}=6 \mathrm{~ms}$ (right). The images are characteristic of softwood species. Indeed, it is possible to detect the typical alternation of lighter and darker bands that corresponds to the alternation of earlywood and latewood.

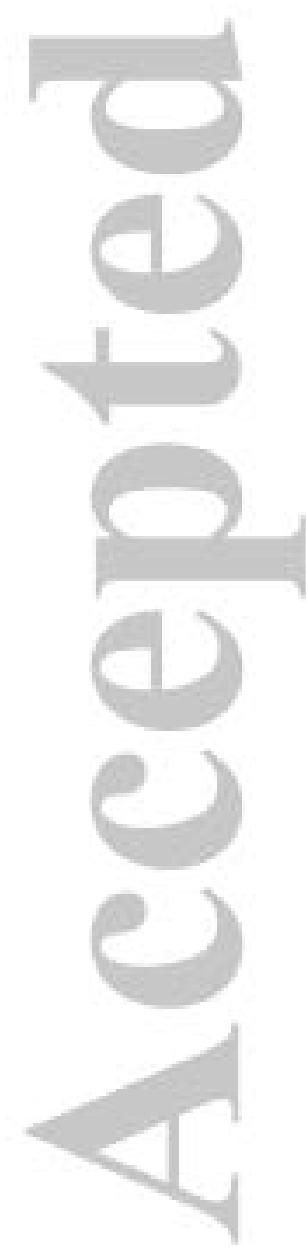



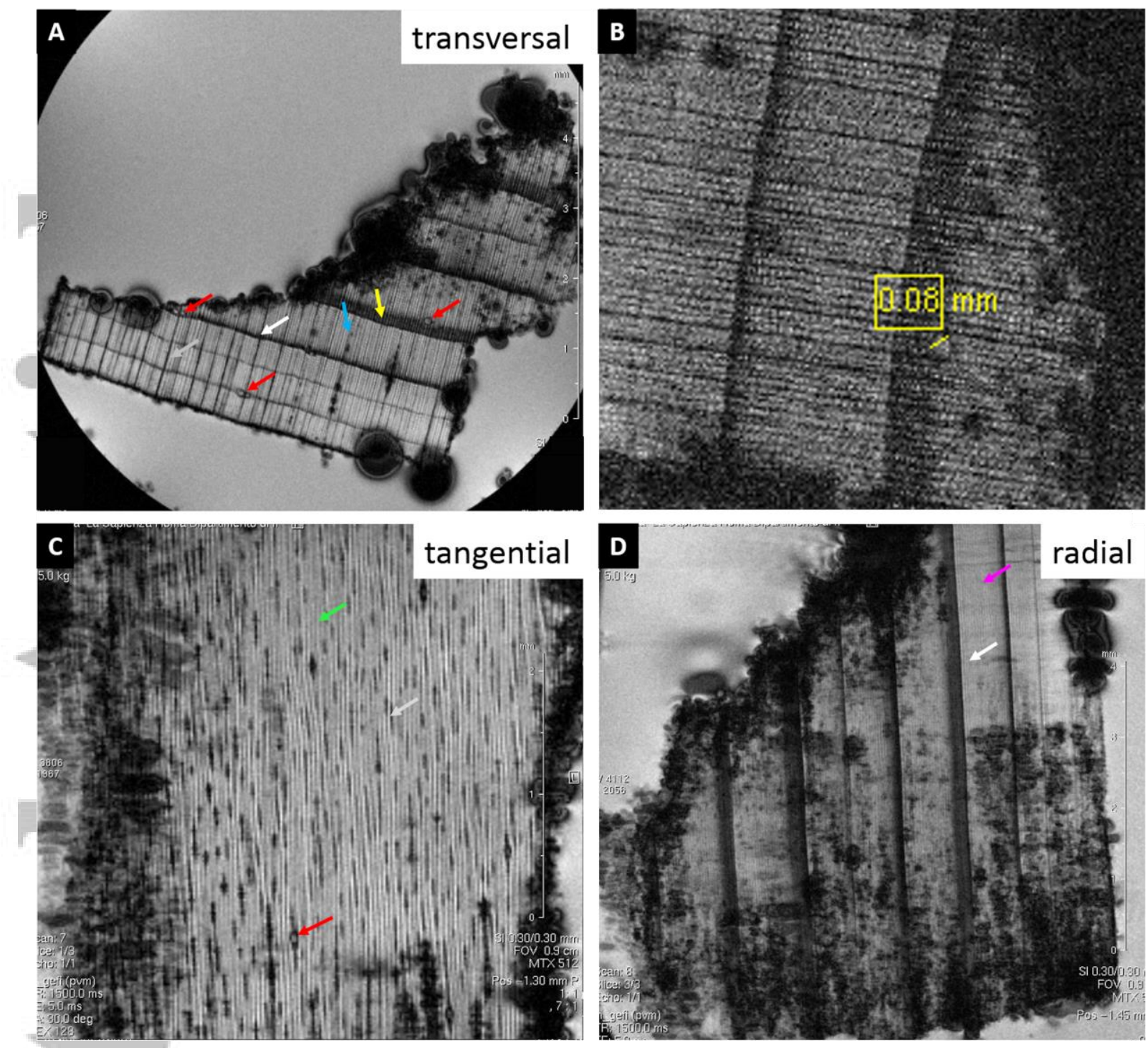

Fig.5 Transversal a), tangential c), and radial d) -view $\mathrm{T}_{2} *$-weighted images of an archaeological waterlogged wood specimen (from an ancient port in Naples of the 5th century AD). Different kinds of microstructures are recognizable. In Fig. 5a): earlywood (blue arrow), latewood (yellow arrow), resin canals (red arrows), annual ring limit (white arrow), and ray (grey arrow). In Fig. 5b) a zoomed portion of the image shown in Fig. 5a) is displayed with the measured diameter of the resin canal around $0.08 \mathrm{~mm}$. In Fig. 5c):

tangential resin canal (red arrow), the tangential section of tracheids, fibers and axial parenchyma of wood (green arrow), and the rays that seem unicellular i.e. composed by a single line of cells (grey arrow). Fig. 5d): annual ring limit (white arrow), the radial section of

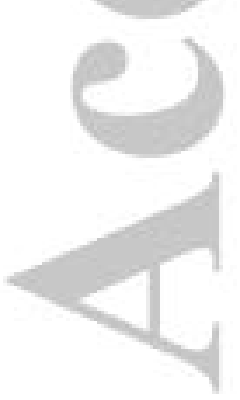
tracheids, fibers and parenchyma (pink arrow). 


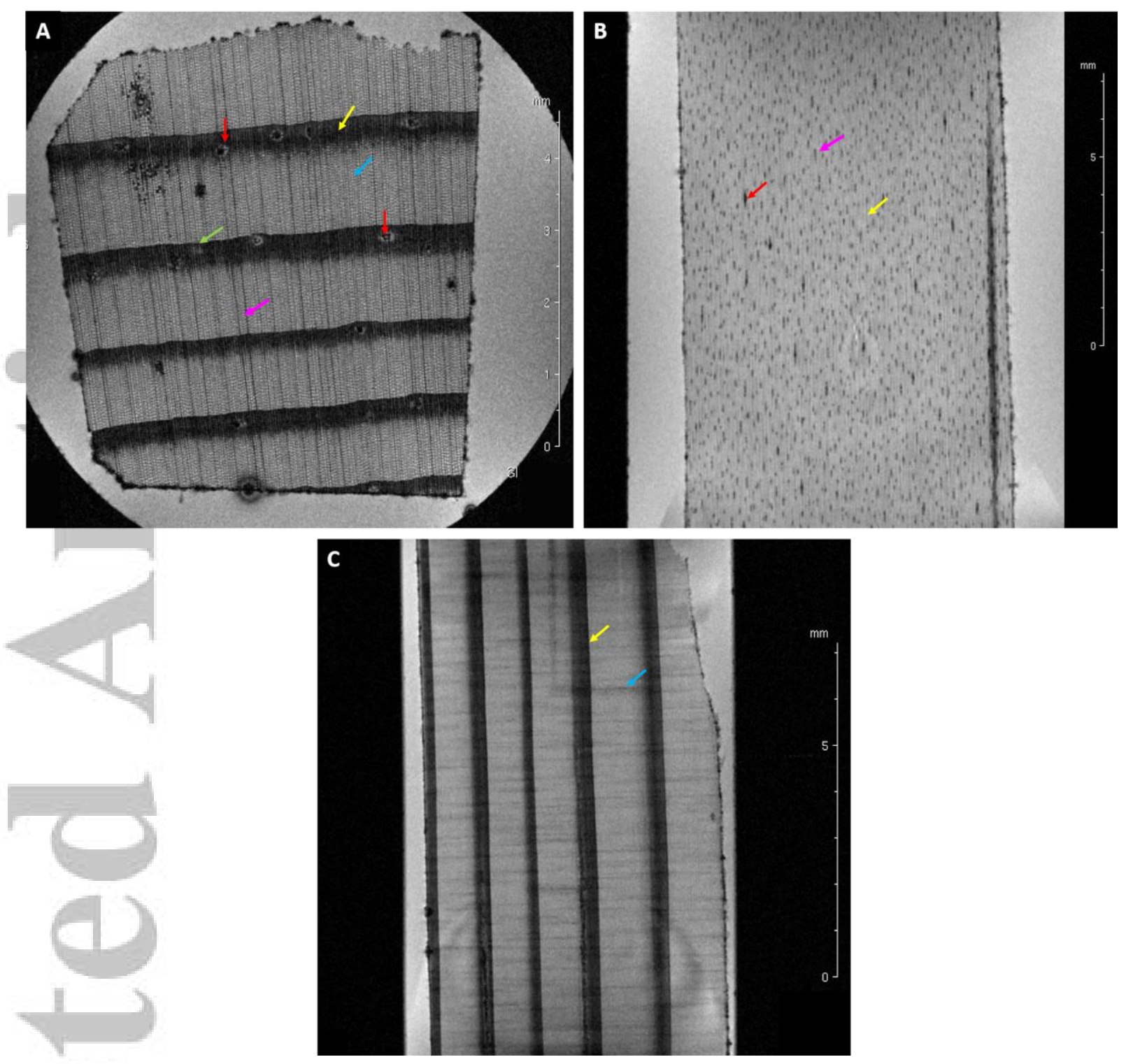

Fig. 6 Transversal a), tangential b), and radial c) -view $T_{2}{ }^{*}$-weighted images of water imbibed Pine. The passage from earlywood (blue arrow) to latewood (yellow arrow) is abrupt as much as in the archaeological wood sample in Fig. 5. Different kinds of microstructures are recognizable. In Fig. 6a): resin canals (red arrows), ray (pink arrow) and annual ring limit (green arrow). In Fig. 6b): tangential resin canal (red arrow), unicellular ray (pink arrow), and the length of tracheids, fibers and axial parenchyma (yellow arrow). In Fig. 6c): the annual ring limit (yellow arrow) and the shadow of the ray (blue arrow). 

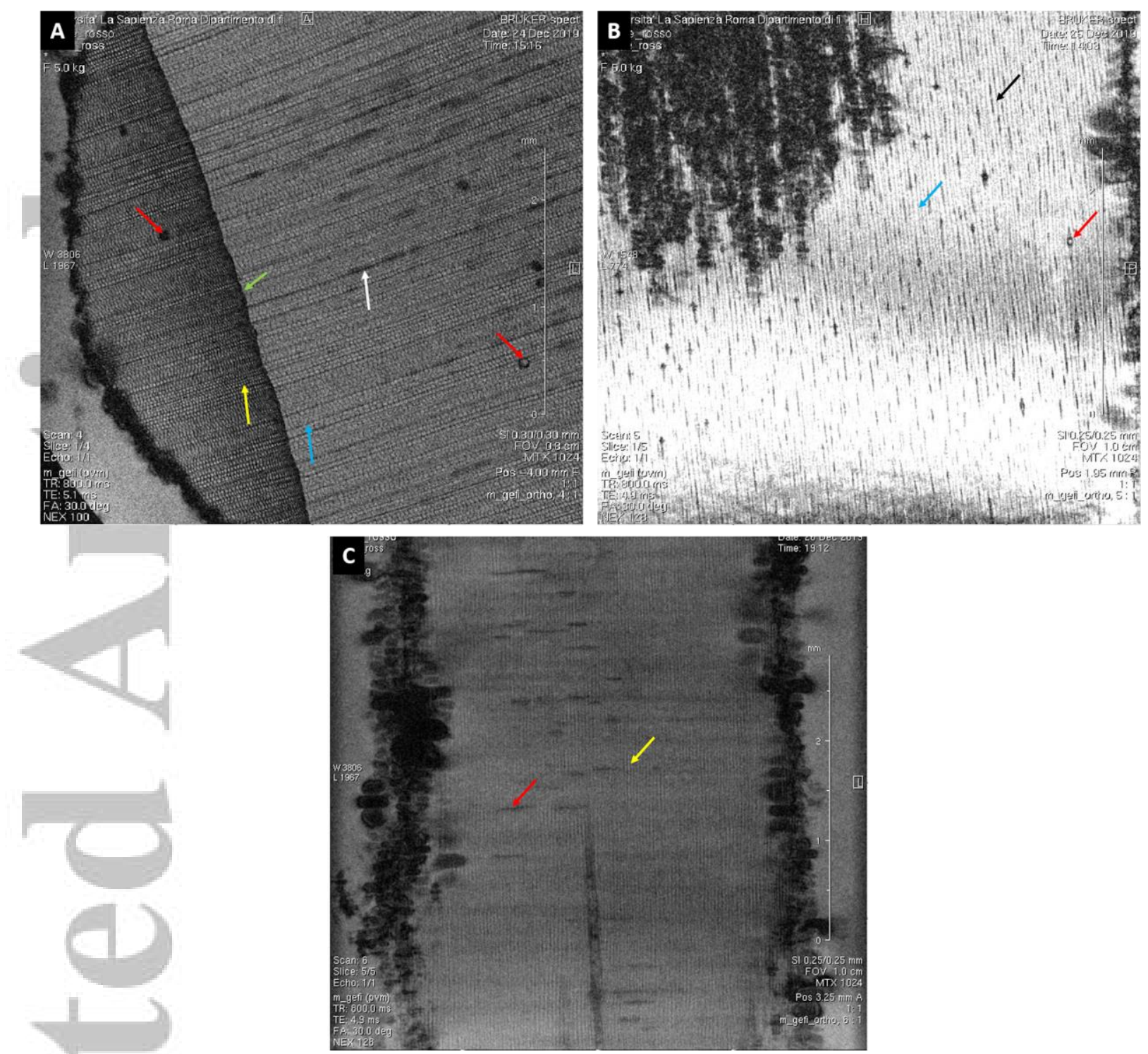

Fig. 7 Transversal a), tangential b), and radial c) -view $\mathrm{T}_{2}{ }^{*}$-weighted images of water imbibed Spruce. The in-plane resolution is equal to $10 \mathrm{x} 10 \mu \mathrm{m}^{2}$. Fig. $7 \mathrm{a}$ ): the passage from earlywood (blue arrow) to latewood (yellow arrow) is gradual. It is possible to identify: resin canals (red arrows), ray (white arrow) and annual ring limit (green arrow). Fig. 7b): the thickness of the ray (black arrow) that seems unicellular, tangential resin canal (red arrow) and the length of tracheids, fibers and axial parenchyma (blue arrow). Fig. 7c) the shadow of the ray (red arrow), and the radial section of tracheids, fibers, and parenchyma (yellow arrow). 

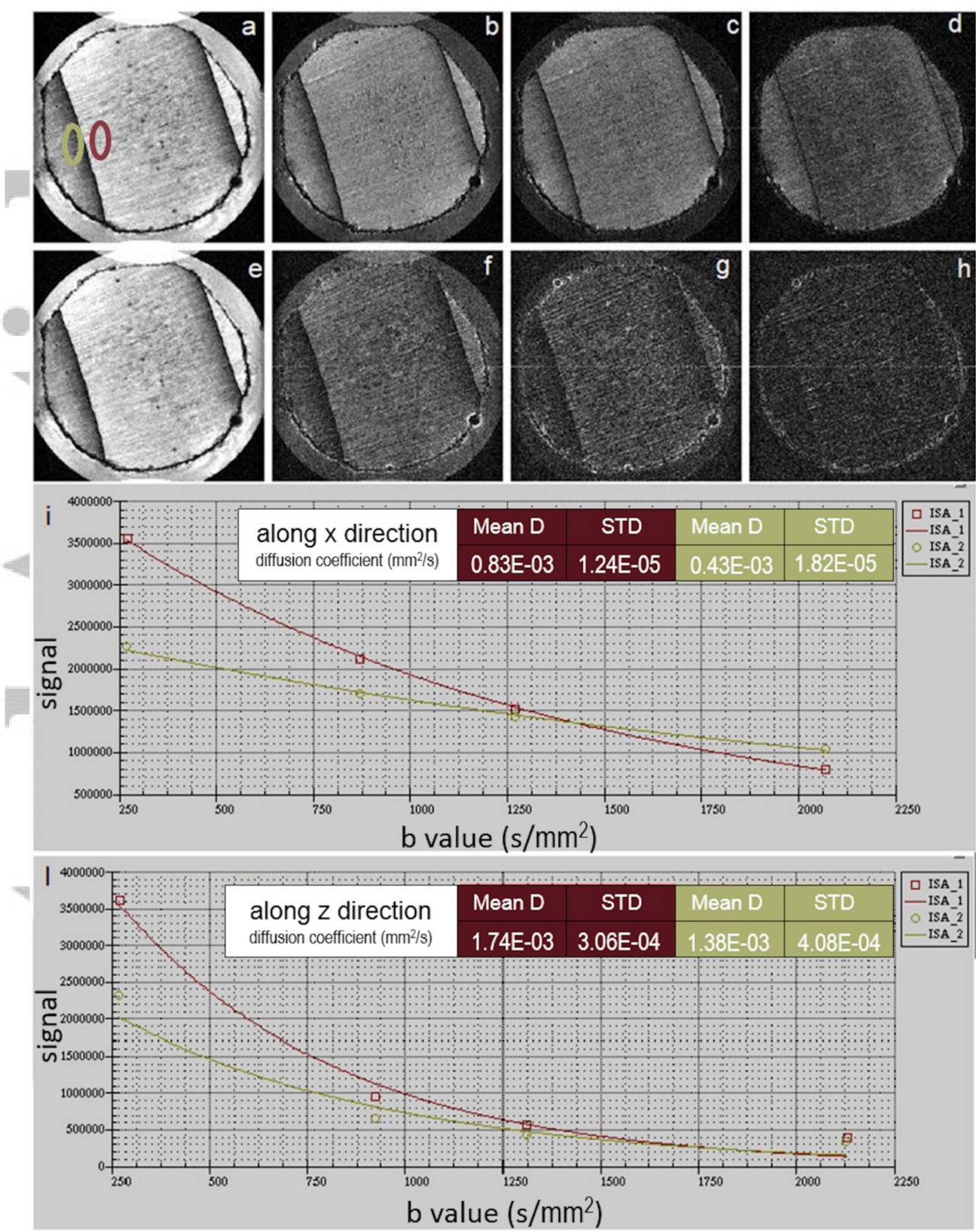

Fig. 8 Axial/Transversal-view diffusion-weighted images of Spruce. Images a), b) c) and d) are obtained using $b=260,860,1260,2060 \mathrm{~s} / \mathrm{mm}^{2}$ with diffusion gradient $\mathrm{g}$ along $\mathrm{x}$ direction, while images e), f), g) and h) are obtained using $b=260,960,1300,2160 \mathrm{~s} / \mathrm{mm}^{2}$ with $g$ along z-direction. Figures i) and 1 ) show the NMR signal decay as a function of $b$ values used to quantify diffusion coefficient along $\mathrm{x}$ and $\mathrm{z}$-direction in the two different wood regions of latewood and earlywood highlighted as a yellow and red circle, respectively. 


\section{Grafical Abstract}

High-resolution multiparametric MRI of contemporary and waterlogged archaeological wood

Silvia Capuani*, Valeria Stagno, Mauro Missori, Laura Sadori, Sveva Longo.

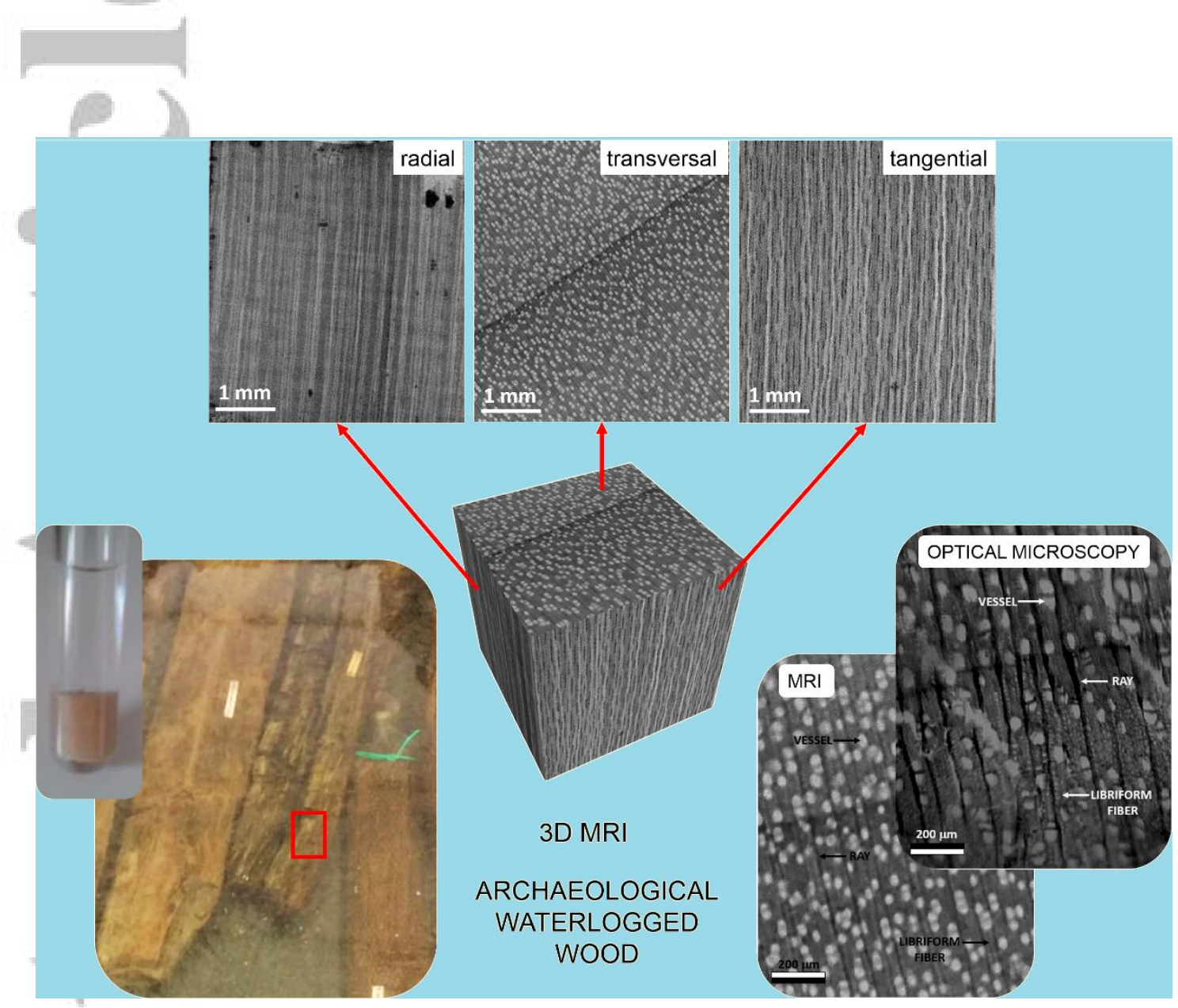

MRI on three different present-day wood samples and an archaeological wood specimen were presented and discussed. Although the spatial resolution is still low to perform dendrology for exact identification of the wooden species, NMR images exhibit contrasts that are in excellent agreement with optical histological images. Moreover, multiparametric MRI, provides multiple structural and functional information that allows obtaining more information than just the structural information provided by optical imaging. 